\title{
Análise numérica e geométrica com design construtal da região de transição para um dispositivo do tipo coluna de água oscilante (CAO)
}

Numerical and geometric analysis with constructal design of the transition region for a oscillating water column (OWC) device

\author{
Y. T. B. Lima ${ }^{1}$; L. A. O. Rocha ${ }^{1}$; M. N. Gomes ${ }^{2}$; E. D. Santos ${ }^{3}$; L. A. Isoldi ${ }^{3}$

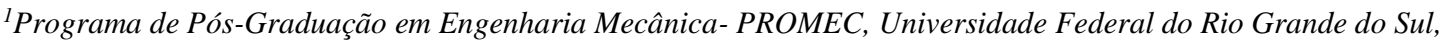 \\ 90050-170,Porto Alegre-RS, Brasil \\ ${ }^{2}$ Instituto Federal do Paraná, Campus Paranaguá, 83215-750, Paranaguá-PR, Brasil \\ ${ }^{3}$ Escola de Engenharia, Universidade Federal do Rio Grande, 96201-900, Rio Grande-RS, Brasil
}

*yuri_vo@hotmail.com

(Recebido em 15 de abril de 2017; aceito em 22 de maio de 2017)

\begin{abstract}
O presente trabalho apresenta um estudo numérico bidimensional sobre a região de transição entre a câmara hidropneumática e a chaminé de um dispositivo conversor do tipo Coluna de Água Oscilante (CAO). O objetivo é maximizar a potência hidropneumática, considerando uma geometria semicircular na região de transição do dispositivo $\mathrm{CAO}$, situada entre a câmara hidropneumática e a chaminé do mesmo. Os resultados são comparados através do valor de potência hidropneumática, para as diferentes configurações geométricas investigadas. O Design Construtal, atrelado ao método de busca exaustiva, foi empregado para definir as dimensões que maximizam a potência hidropneumática. Os graus de liberdade estudados foram o raio (r), a razão entre altura e comprimento da chaminé de saída de ar da câmara $\mathrm{CAO}\left(\mathrm{H}_{2} / \mathrm{l}\right)$ e a razão entre a altura e o comprimento da câmara $\mathrm{CAO}\left(\mathrm{H}_{1} / \mathrm{L}\right)$. O domínio computacional é representado por um tanque de ondas com o dispositivo CAO acoplado. O software FLUENT foi empregado para a solução numérica das equações, baseada no Método dos Volumes Finitos (MVF). A construção da geometria e a geração da malha foram realizadas no software GAMBIT. Na interação entre as fases ar e água foi aplicado o método Volume of Fluid (VOF). Os resultados obtidos mostram que a máxima potência hidropneumática de $190,61 \mathrm{~W}$ é obtida para os graus de liberdade $\mathrm{H}_{1} / \mathrm{L}=0,1346$ e $\mathrm{r}=7,54$, o caso com a mais baixa potência hidropnemática, apresenta 57,73W. Este resultado mostra um ganho de $69,90 \%$ em relação ao caso com menor desempenho de potência hidropneumática.

Palavras-chave: Design Construtal, Coluna de Água Oscilante (CAO), Região de Transição
\end{abstract}

The present work presents a two-dimensional numerical study on the transition region between the hydropneumatic chamber and the chimney of a Oscillating Water Column (OWC) converter. The objective is to maximize the hydropneumatic power, considering a semicircular geometry in the transition region of the OWC device, located between the hydropneumatic chamber and the chimney of the same. The results are compared through the hydropneumatic power values for the different geometric configurations investigated. The Design Constructal, coupled with the exhaustive search method, was used to define dimensions that maximize hydropneumatic power. The degrees of freedom studied were the radius (r), the ratio between height and length of the air outlet chimney of the OWC chamber $\left(\mathrm{H}_{2} / \mathrm{l}\right)$ and the ratio between the height and the length of the OWC chamber $\left(\mathrm{H}_{1} / \mathrm{L}\right)$. The computational domain is represented by a wave tank with the OWC device coupled. The FLUENT software was used for the numerical solution of the equations, based on the Finite Volume Method (FVM). The construction of the geometry and the generation of the mesh were realized in the software GAMBIT. In the interaction between the air and water phases the Volume of Fluid (VOF) method was applied. The results show that the maximum hydropneumatic power of $190.61 \mathrm{~W}$ is obtained for the degrees of freedom $\mathrm{H}_{1} / \mathrm{L}=0.1346$ and $\mathrm{r}=7.54$, the case with the lowest hydropneumatic power, presents $57.73 \mathrm{~W}$. The results show a gain of $69.90 \%$ in relation to the case with lower performance of hydropneumatic power.

Keywords: Constructal Design, Oscillating Water Column (OWC), Transition Region 


\section{INTRODUÇÃO}

Um dos maiores desafios para o desenvolvimento tecnológico atualmente passa pelo equilíbrio entre a inovação e a sustentabilidade dos recursos utilizados. Neste sentido, muitos países estão investindo em pesquisas e exploração de novas formas de energia.

Algumas possibilidades são: energia solar, energia eólica e energia dos oceanos. E uma dessas fontes de energia que tem merecido destaque, é a transformação da energia contida nos oceanos em energia elétrica [1].

A energia contida nos oceanos pode ter origens diferentes, o que gera diferentes classificações. Para realizar a transformação de energia das ondas regulares em energia elétrica, existem diversos tipos de conversores com diferentes classificações. Os dispositivos podem ser classificados quanto ao seu local de instalação: dispositivos costeiros (onshore), em regiões com profundidades de até $20 \mathrm{~m}$, e que são geralmente presos ao fundo; dispositivos próximos à costa (nearshore), situados em regiões com profundidades entre 20 e $50 \mathrm{~m}$, podem ser presos ao fundo ou flutuantes; dispositivos afastados da costa (offshore), situados em regiões com profundidades maiores que 50 $\mathrm{m}$, são geralmente flutuantes [1].

A classificação dos conversores de energia das ondas quanto ao seu princípio de funcionamento pode ser: Coluna de Água Oscilante (CAO), Galgamento e Corpos Oscilantes [1]. Entre as diferentes tecnologias de conversão de energia das ondas regulares em energia elétrica, a que tem mais se destacado, em número de projetos, é a baseada no princípio CAO.

No presente trabalho, o objetivo geral é através da modelagem computacional, do método Design Construtal e do método de busca exaustiva, simular numericamente o princípio de funcionamento do conversor tipo CAO.

A avaliação da influência que a região de transição semicircular possui sobre o escoamento do ar pelo interior do dispositivo CAO é comparada com os resultados já obtidos nas pesquisas desenvolvidas em Lima et al. (2004) [2]. Assim, visando maximizar a conversão de energia das ondas regulares em energia elétrica.

Considerando uma geometria bidimensional, as restrições para o problema são: área de entrada da câmara hidropneumática $\left(A_{E}\right)$ e a área da câmara hidropneumática somada à área da chaminé $\left(A_{T}\right)$, as quais são mantidas constantes.

O método Design Construtal é baseado na Teoria Construtal desenvolvida por Bejan (2012, 2013) $[4,5]$. A teoria Construtal explica deterministicamente como são geradas as formas de fluxo na natureza (bacias hidrográficas, pulmões, circulação atmosférica, forma de animais, tecidos vasculares, etc.) com base em um princípio evolutivo de acesso, de forma fácil, ao fluxo no tempo.

Existe uma gama de trabalhos os quais se utilizam do método Design Construtal para a otimização geométrica de dispositivos conversores de energia das ondas do mar em energia elétrica, dentre eles destacam-se as pesquisas desenvolvidos por: Lima et al. $(2004,2014)[2,14]$; Gomes (2014) [3], Gomes et al. (2009, 2012, 2013) [6, 7, 8]; Lopes et al. (2012) [9]; Oliveira et al. (2012) [10]; Espinel et al. (2014) [11, 12]; Espinel (2015) [13].

Para modelagem computacional, do princípio de funcionamento do conversor CAO, o domínio computacional (dispositivo CAO acoplado a um tanque de ondas) é discretizado no software GAMBIT $^{\circledR}$, enquanto as simulações numéricas foram desenvolvidas utilizando-se um código de dinâmica de fluidos, FLUENT ${ }^{\circledR}$, baseado no Método de Volumes Finitos (MVF) $[15,16]$. O modelo multifásico Volume of Fluid (VOF) é aplicado no tratamento da interação água-ar [17, 18, 19].

\section{MATERIAL E MÉTODOS}

\subsection{Princípio de funcionamento}

Os dispositivos do tipo Coluna de Água Oscilante são estruturas ocas parcialmente submersas, abertas para o mar abaixo da superfície livre da água como pode ser observado na Figura 1. O processo de transformação de energia segue duas fases: quando uma onda entra no dispositivo, o ar que se encontrava dentro dela é forçado a passar por uma turbina, como consequência direta do 
aumento de pressão na câmara hidropneumática. Quando a onda deixa o dispositivo, o ar passa novamente na turbina, desta vez no sentido inverso, dada a pressão inferior no interior da câmara hidropneumática.

Para se ter o aproveitamento do escoamento nos dois sentidos (compressão e descompressão), normalmente são utilizadas turbinas do tipo Wells, as quais apresentam a propriedade de manterem o mesmo sentido de rotação das suas pás, independente do sentido do escoamento. $\mathrm{O}$ conjunto turbina e gerador é o responsável pela geração de energia elétrica [1].

A câmara CAO é o dispositivo mais avançado em quantidade e duração de protótipos testados no mar, há inclusive algumas centrais piloto instaladas em Portugal e Escócia. O mecanismo CAO, pode ser representado por quatro componentes principais: câmara hidropneumática, chaminé, turbina e gerador.

Com relação as suas dimensões, as mesmas estão apropriadas para o tipo de onda local $[3,20]$. É importante, no projeto de dispositivos do tipo CAO, conhecer a potência da onda incidente e as forças que atuam sobre ele, assim é possível adequar a geometria do equipamento à quantidade de energia que se pretende extrair.

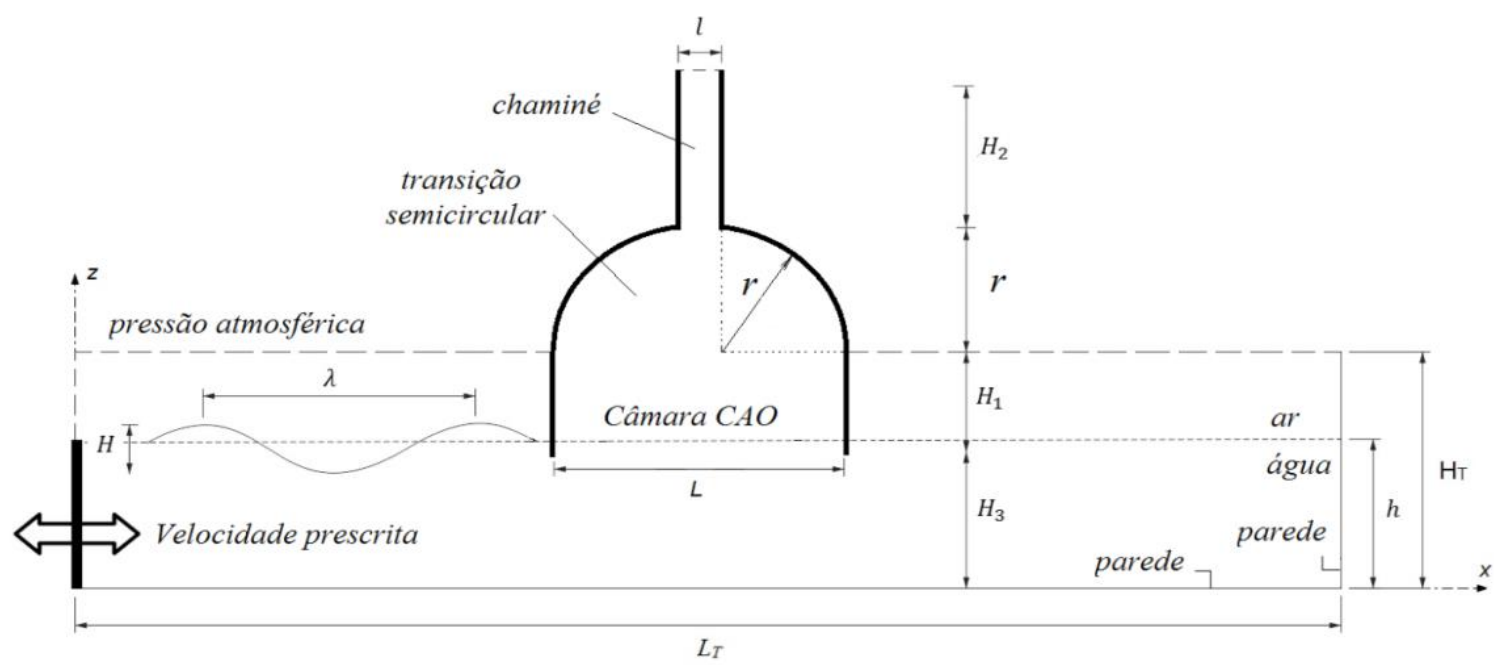

Figura 1: Representação esquemática do domínio computacional.

\subsection{Domínio Computacional}

O domínio computacional bidimensional apresentado é composto pelo dispositivo CAO inserido em um tanque de ondas. As dimensões do dispositivo são a altura da câmara $\left(H_{l}\right)$, profundidade de submersão do dispositivo $\left(H_{3}\right)$, a altura da chaminé $\left(H_{2}\right)$, comprimento da chaminé $(l)$, comprimento da câmara $(L)$ e altura da região de transição $(r)$ apresentado na Figura1.

Para representar o problema numa escala adequada no domínio computacional é necessário o conhecimento de algumas características do clima de ondas, como: período $(T)$ e altura $(H)$ para a onda gerada, com profundidade ou nível de água $(h)$, assim o comprimento $\left(L_{T}\right)$ e a altura $\left(H_{T}\right)$ do tanque de ondas podem ser definidas utilizando estes parâmetros citados [7].

A profundidade de propagação da onda é representada por $h$. Para o comprimento do tanque é preciso considerar o comprimento da onda $(\lambda)$. É recomendado que o comprimento do tanque seja, aproximadamente, cinco vezes maior que o comprimento da onda. Assim garante-se a simulação numérica da onda sem efeito de reflexão por um tempo razoável de propagação. A altura do tanque de ondas deve ser a profundidade de água preenchida no tanque mais três vezes a altura da onda [3].

\subsection{Domínio Computacional}

No trabalho realizado, a onda incidente é gerada no lado esquerdo do tanque de ondas, local onde o gerador de ondas numérico é posicionado, como pode ser observado na Figura 1. O 
problema é tratado em escala real e sendo consideradas as seguintes características: $T=5,0 \mathrm{~s}, H=$ $1,0 \mathrm{~m}, \lambda=37,6 \mathrm{~m}, h=10,0 \mathrm{~m}, L_{T}=188 \mathrm{~m}, H_{T}=13 \mathrm{~m}$.

Para a geração da onda regular, a Metodologia Função é aplicada [21]. Esta metodologia consiste na criação de uma função definida pelo usuário, UDF (User Define Function), no FLUENT.

A UDF separa o perfil de velocidade da onda em componentes horizontal e vertical, com base na Teoria de Stokes de $2^{\mathrm{a}}$ ordem [2]. Assim, as equações impostas na região de entrada são dadas por [22]:

$$
\begin{aligned}
& u=\frac{H}{2} g k \frac{\cosh (k z+k h)}{\omega \cosh (k h)} \cos (k x-\omega t)+\frac{3}{4}\left(\frac{H}{2}\right)^{2} \omega k \frac{\cosh 2 k(k+z)}{\sinh ^{4}(k h)} \cos 2(k x-\omega t) \\
& v=\frac{H}{2} g k \frac{\operatorname{senh}(k z+k h)}{\omega \cosh (k h)} \operatorname{sen}(k x-\omega t)+\frac{3}{4}\left(\frac{H}{2}\right)^{2} \omega k \frac{\operatorname{senh} 2 k(k+z)}{\operatorname{senh}^{4}(k h)} \operatorname{sen} 2(k x-\omega t)
\end{aligned}
$$

onde $H$ é a altura da onda incidente $(\mathrm{m}) ; g$ é a aceleração da gravidade $\left(\mathrm{m} / \mathrm{s}^{2}\right) ; k$ é o número de ondas dado por $k=2 \pi / \lambda\left(\mathrm{m}^{-1}\right), h$ é a profundidade $(\mathrm{m}), T$ é o período de onda (s), $\omega$ é a frequência dada por $\omega=2 \pi / T(\mathrm{rad} / \mathrm{s}), x$ é a coordenada que representa a direção principal (m), $t$ é o tempo (s) e $z$ é a coordenada normal $(\mathrm{m})$.

Com relação às outras condições de contorno, na parte superior da superfície lateral esquerda, bem como na superfície superior do tanque e da saída da chaminé da CAO é aplicada uma condição de contorno de pressão atmosférica (vide superfície tracejada na Figura 1).

Nas demais superfícies, são impostas condições de não deslizamento e impermeabilidade com velocidade nula, respectivamente. Com relação às condições iniciais, foi considerado que o fluido está em repouso, com profundidade $h=10,0 \mathrm{~m}$.

\subsection{Design Construtal}

O Design Construtal é um método que relacionando graus de liberdade, restrições e função objetivo, busca avaliar a influência da geometria no desempenho de um determinado sistema [5]. Se um método de busca exaustiva for associado ao método Design Construtal, todas as geometrias possíveis serão testadas e então será possível determinar a geometria ótima que maximiza o desempenho do sistema.

A Lei Construtal mostra qual é a tendência ou caminho que a natureza assume para obter o melhor desempenho no que se propõe e com isso permite obter uma melhor condição de fluxo. Assim, é possível projetar processos de forma orgânica e dinâmica para obter melhores ou mais eficientes resultados [5].

Neste trabalho, a função objetivo é maximizar a potência hidropneumática do conversor CAO. As restrições para o problema são: área de entrada da câmara hidropneumática $\left(A_{E}\right)$, área total da câmara CAO $\left(A_{T}\right)$, as mesmas são mantidas constantes, e podem ser calculadas através das seguintes relações, respectivamente:

$$
A_{E}=H_{1} L
$$

$$
A_{T}=H_{1} L+H_{2} l
$$

Um critério é adotado para a definição da restrição da área de entrada da câmara do dispositivo CAO $\left(A_{E}\right)$, considera-se a situação inicial em que o comprimento do dispositivo CAO $(L)$ é igual ao comprimento da onda incidente $(\lambda)$ e que a altura do dispositivo CAO $\left(H_{l}\right)$ é igual à altura da onda incidente $(H)$ [3]. 
E, para determinar a restrição da área da câmara hidropneumática somada à área da chaminé $\left(A_{T}\right)$, é definido que $A_{E}$ representa $70 \%$ de $A_{T}$, ou seja: $A_{T}=1,43 A_{E}$. Neste estudo tem-se que $A_{\mathrm{E}}=$ $37,6 \mathrm{~m}^{2}$ e $A_{T}=53,76 \mathrm{~m}^{2}$.

Na presente investigação os graus de liberdade analisados são o raio $(r), H_{2} / l$ (razão entre altura e comprimento da chaminé de saída da câmara CAO) e $H_{l} / L$ (razão entre a altura e o comprimento da câmara $\mathrm{CAO}$ ). $\mathrm{O}$ grau de liberdade $H_{3}$ (profundidade de submersão do dispositivo $\mathrm{CAO}$ em relação à superfície livre) é mantido fixo, com $H_{3}=9,5 \mathrm{~m}$, segundo a recomendação obtida por Gomes [3].

Ao variar os graus de liberdade é possível dimensionar a geometria do dispositivo CAO através das seguintes relações:

$$
L=2 r+l
$$

Sendo os comprimentos $L$ e $l$ calculados, respectivamente, por:

$L=\left[\frac{A_{E}}{\left(H_{1} / L\right)}\right]^{1 / 2}$

$l=\left[\frac{A_{T}-A_{E}}{\left(H_{2} / l\right)}\right]^{1 / 2}$

onde as alturas $H_{1}$ e $H_{2}$ são calculadas, respectivamente, por:

$$
\begin{aligned}
& H_{1}=L\left(\frac{H_{1}}{L}\right) \\
& H_{2}=l\left(\frac{H_{2}}{l}\right)
\end{aligned}
$$

Com a variação do grau de liberdade $H_{2} / l$, é possível definir o diâmetro $(l)$ e a altura $\left(H_{2}\right)$ da chaminé de saída da câmara CAO, através da Equação (8) e da Equação (9), respectivamente. Assim o raio $(r)$ da região de transição é também variado, e calculado por:

$$
r=\left(\frac{L-l}{2}\right)
$$

\subsection{Modelo Volume of Fluid (VOF)}

O modelo Volume of Fluid (VOF) consiste em reproduzir a interação entre os fluidos envolvidos na simulação numérica do dispositivo do tipo CAO, que são o ar e a água neste caso. Neste modelo todas as fases são definidas e o volume ocupado por uma fase não pode ser ocupado por outra fase.

O modelo se utiliza do conceito de fração de volume $(\alpha)$ para encontrar como as fases são distribuídas ao longo do volume de controle, como mais do que uma fase não pode ocupar o mesmo volume ao mesmo tempo, e, por conseguinte, a fração volumétrica em cada célula deve ser a soma de $\alpha_{a r}$ e $\alpha_{\text {água }}$ e sempre ser igual a um $\left(\alpha_{a r}+\alpha_{\text {água }}=1\right)$.

No modelo VOF, as equações de continuidade e quantidade de momento são aplicadas a todos os fluidos que compõem o escoamento, e a fração de volume de cada fluido em cada célula é 
considerada em todo o domínio computacional através da equação de fração volumétrica. As equações de continuidade, fração volumétrica e quantidade de momento são, respectivamente, definidas por:

$$
\begin{aligned}
& \frac{\partial \rho}{\partial t}+\nabla \cdot(\rho \vec{v})=0 \\
& \frac{\partial(\alpha)}{\partial t}+\nabla \cdot(\alpha \vec{v})=0 \\
& \frac{\partial}{\partial t}(\rho \vec{v})+\nabla \cdot(\rho \overrightarrow{v v})=-\nabla p+\nabla(\mu \tau)+\rho g
\end{aligned}
$$

onde: $\rho$ é a massa específica do fluido $\left(\mathrm{kg} / \mathrm{m}^{3}\right), t$ é o tempo (s), $v$ é o vetor velocidade do escoamento $(\mathrm{m} / \mathrm{s}), p$ é a pressão estática $\left(\mathrm{N} / \mathrm{m}^{2}\right), \mu$ é a viscosidade $(\mathrm{kg} / \mathrm{m} \cdot \mathrm{s}), \tau$ é o tensor de tensões $\left(\mathrm{N} / \mathrm{m}^{2}\right)$ e g é a aceleração da gravidade $\left(\mathrm{m} / \mathrm{s}^{2}\right)$.

Uma vez que as equações de conservação de massa e quantidade de movimento são resolvidas para a mistura de ar e água, é necessário calcular valores médios para a massa específica e a viscosidade, respectivamente [25]:

$$
\begin{aligned}
& \rho=\alpha_{\text {agua }} \rho_{\text {agua }}+\alpha_{\text {ar }} \rho_{\text {ar }} \\
& \mu=\alpha_{\text {agua }} \mu_{\text {agua }}+\alpha_{\text {ar }} \mu_{\text {ar }}
\end{aligned}
$$

\subsection{Modelo Numérico}

Para a solução numérica das equações de conservação da massa e quantidade de movimento, foi empregado um código baseado no Método de Volumes Finitos - MVF [15]. Para todas as simulações, foi considerado o esquema de advecção UPWIND para tratamento dos termos advectivos, enquanto a discretização espacial para a pressão foi realizada através do método PRESTO e o método GEO-RECONSTRUCTION para a fração volumétrica.

Quanto ao acoplamento pressão-velocidade, foi empregado o método PISO. Para a solução do sistema de equações gerado, após a discretização, é utilizado o método Gauss Seidel com multigrid algébrico. Maiores detalhes sobre a metodologia numérica podem ser obtidos em Patankar (1980) [26] e Versteeg (2007) [27].

Todas as soluções foram realizadas usando um computador Intel Core i7 com $8.0 \mathrm{~Gb}$ de RAM, utilizando processamento em série. O tempo de processamento de cada simulação foi de aproximadamente $4 \mathrm{~h}$.

\subsection{Verificação do Modelo Computacional}

O modelo VOF tem sido empregado para simulações numéricas de conversores de energia das ondas do mar. A validação desta metodologia pode ser encontrada na literatura como em Gomes et al. (2009, 2012) [17, 21], Liu et al. (2008) [18, 29], Ramalhais (2011) [19], Horko (2007) [28] e Gomes (2010) [30].

A verificação da presente solução com o modelo VOF consiste em gerar a onda em um tanque de ondas e observar a sua variação na superfície livre da água, ou seja, a sua amplitude, para posteriormente comparar com a equação analítica para a variação da superfície livre da água em um dado ponto do tanque de ondas. 
A comparação da solução numérica com a solução analítica foi proposta por [23], a equação analítica da superfície livre é definida por:

$$
\eta(x, t)=A \cos (k x-\omega t)+\frac{A^{2} k \cosh (k h)}{4 \operatorname{senh}^{3}(k h)}[2+\cosh (2 k h)] \cos 2(k x-\omega t)
$$

onde: $A$ é a amplitude da onda (m), dado por $H / 2, x$ é a posição $(\mathrm{m}), t$ é a variação do tempo (s), $k$ e $\omega$ são respectivamente o número de onda $\left(\mathrm{m}^{-1}\right)$ e a frequência da onda $(\mathrm{rad} / \mathrm{s})$.

Na Figura 2, foram comparados os resultados obtidos numericamente com os obtidos pela Equação (16) na posição $x=22,5 \mathrm{~m}$. A verificação da solução numérica foi realizada no intervalo entre 20 e 30 s onde a onda numérica está estável e ainda não há reflexão da mesma.

A diferença relativa entre as soluções numérica e analítica foi medida instantaneamente, e a média das diferenças foi $2,61 \%$, onde o valor mínimo obtido foi $0,0019 \%$ e o valor máximo $5,86 \%$. Estes resultados indicam a acurácia do modelo.

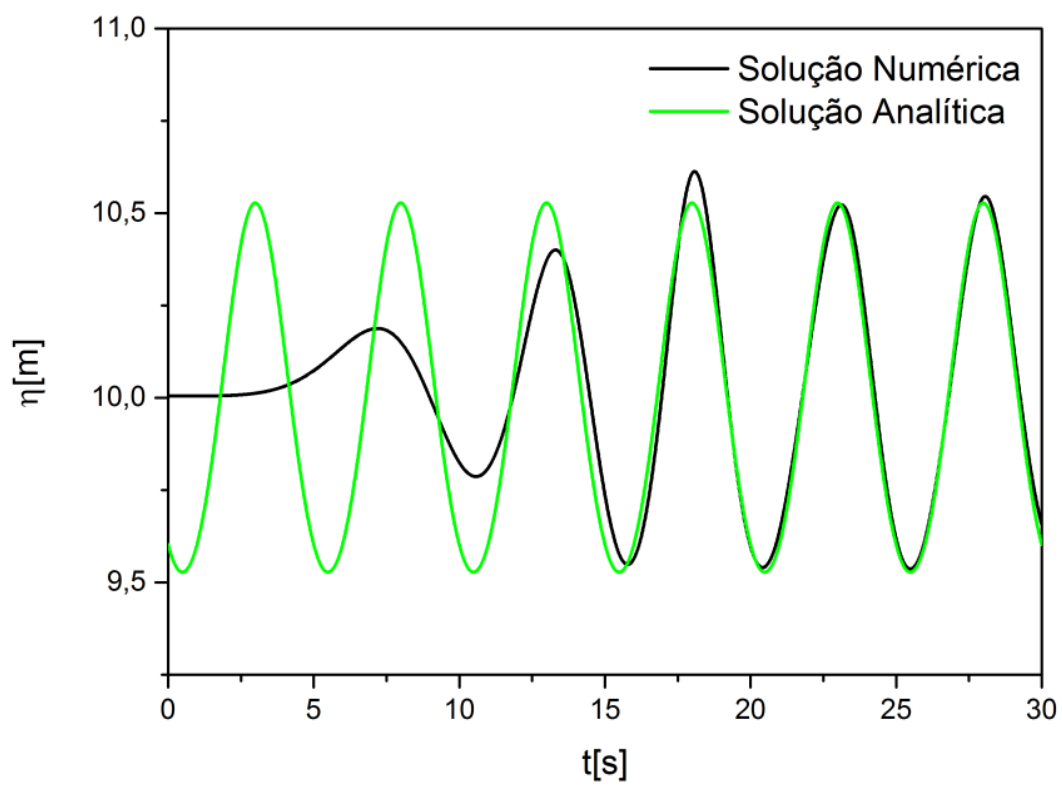

Figura 2: Elevação da superfície livre da água ao longo do tempo.

Para a geração da malha foi adotada uma estratégia que tem como objetivo construir uma malha mais refinada em determinadas regiões de interesse no domínio computacional, como a superfície livre por exemplo. Essa metodologia é baseada na técnica de malhas stretched [31]. Para completar o domínio computacional, quadriláteros com $0,1 \mathrm{~m}$ de lado foram utilizados na discretização do dispositivo CAO.

Neste trabalho foram avaliadas a vazão mássica (na saída da chaminé), a pressão (no interior da câmara), a eficiência e a potência hidropneumática. Os valores médios foram calculados empregando a média aritmética para problemas transientes Root Mean Square (RMS) [32]:

$$
X=\sqrt{\frac{1}{T} \int_{0}^{T} x^{2} d t}
$$

onde $x$ representa a grandeza que se deseja calcular a média RMS. 
A potência hidropneumática é calculada por [33]:

$$
P_{h i d}=\left(P_{a r}+\frac{\rho_{a r} v_{a r}{ }^{2}}{2}\right) \frac{\dot{m}}{\rho_{a r}}
$$

onde: $P_{a r}$ é a pressão estática na chaminé do dispositivo CAO (Pa), $\rho_{a r}$ é a densidade do ar $\left(\mathrm{kg} / \mathrm{m}^{3}\right), \dot{m}$ é a taxa de vazão mássica atravessando a chaminé $(\mathrm{kg} / \mathrm{s}), v_{a r}$ é a velocidade do ar na chaminé $(\mathrm{m} / \mathrm{s})$ dada por:

$$
v_{a r}=\frac{\dot{m}}{A \rho_{a r}}
$$

onde: $A$ representa uma área em corte transversal da chaminé $\left(\mathrm{m}^{2}\right)$. Como o estudo realizado é bidimensional a terceira dimensão é considerada unitária.

As expressões que determinam a pressão estática, pressão total, vazão mássica, eficiência e potência da onda incidente podem ser encontradas em [3].

\section{RESULTADOS E DISCUSSÃO}

Ao todo foram analisadas trinta e sete configurações geométricas para o caso em que a região de transição é na forma semicircular. Todas as dimensões foram calculadas através das Equações (5-10) onde somente o raio $r, H_{1} / L$ e $H_{2} / l$ variam.

Considerando os resultados apresentados na Tabela 1 ,Figura 3 e Figura 4, observa-se que a variação da dimensão do raio da transição $(r)$ é responsável pelo aumento de cerca de $64 \%$ na potência hidropneumática, afetando diretamente a configuração geométrica do dispositivo.

Tabela 1: Resultados com melhor desempenho para todos os valores de $H_{1} / L, H_{2} / l$ e $r$.

\begin{tabular}{ccccccc}
\hline $\mathbf{H}_{\mathbf{1}} / \mathbf{L}$ & $\mathbf{H}_{2} / \mathbf{l}$ & $\begin{array}{c}\text { Raio } \\
{[\mathbf{m}]}\end{array}$ & $\begin{array}{c}\text { Vazão Mássica } \\
{[\mathbf{K g} / \mathbf{s}]}\end{array}$ & $\begin{array}{c}\text { Pressão } \\
{[\mathbf{P a}]}\end{array}$ & $\begin{array}{c}\text { Potência } \\
\text { Hidropneumática }[\mathbf{W}]\end{array}$ & $\begin{array}{c}\text { Eficiência } \\
{[\%]}\end{array}$ \\
\hline 0,05 & 6 & 11,72 & 12,36 & 43,55 & 109,22 & 34,15 \\
0,09 & 6 & 9,42 & 14,71 & 53,35 & 167,34 & 57,37 \\
0,13 & 6 & 7,54 & 15,28 & 58,01 & 190,61 & 66,74 \\
0,20 & 6 & 6,00 & 14,71 & 58,29 & 182,45 & 63,51 \\
0,30 & 6 & 4,75 & 13,48 & 53,74 & 157,82 & 56,27 \\
0,45 & 6 & 3,73 & 11,90 & 47,40 & 124,87 & 45,29 \\
0,68 & 6 & 2,89 & 10,30 & 41,26 & 93,16 & 33,93 \\
1,02 & 6 & 2,21 & 8,75 & 34,88 & 67,31 & 24,13 \\
\hline
\end{tabular}

A variação do grau de liberdade $H_{2} / l$ não se mostra tão evidente, uma vez que a Tabela 1 mostra os resultados com melhor desempenho para as oito variações de $H_{l} / L$, a justificativa para os valores ficarem todos concentrados no mesmo ponto é que para esse grau de liberdade $\mathrm{H}_{2}=$ $9,83 \mathrm{~m}$ e $l=1,64 \mathrm{~m}$ é o maior valor para a altura do tanque e o menor valor para a largura do mesmo, onde assim concentra-se a maior quantidade de pressão, essa que influência diretamente na fórmula da potência hidropneumática (Equação 18).

$\mathrm{O}$ valor do grau de liberdade $H_{1} / L$ se mostrou o esperado, valor esse definido em Lima et al. [2], onde se tinha o mesmo fixo. Lembrando que este trabalho é uma continuação do trabalho realizado em Lima et al. [2] e que a área da chaminé do dispositivo permanece constante em todos os casos estudados.

Observa-se que pequenas variações na dimensão dos três graus de liberdade analisados são suficientes para causar um aumento significativo na eficiência da configuração geométrica proposta, evidenciando a pertinência desta proposta de estudo.

Os resultados obtidos mostram que a máxima potência hidropneumática de 190,61 W é obtida para os graus de liberdade $H_{l} / L=0,1346, H_{2} / l=6$ e $r=7,54 \mathrm{~m}$. Em contrapartida o resultado com 
a menor potência hidropneumática se teve com a configuração de $H_{l} / L=1,0225, H_{2} / l=5$ e $r=$ $2,13 \mathrm{~m}$, onde se obteve um valor de 57,73 W. Este resultado mostra um ganho de 69,71\% em relação ao caso com menor desempenho de potência hidropneumática.

De uma maneira geral, é possível observar que estas variações geométricas na região de transição são pertinentes, uma vez que conduzem a melhores aproveitamentos da potência da onda incidente.

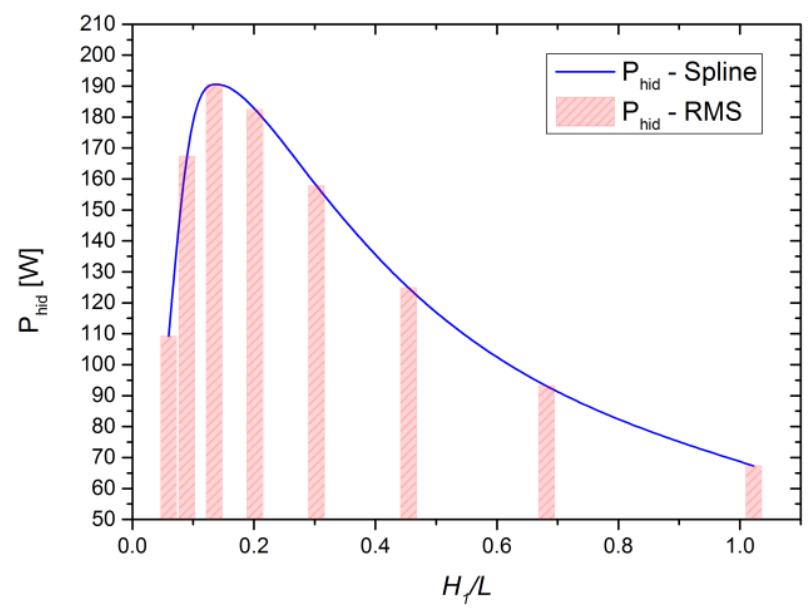

Figura 3: Valores de potência hidropneumática para $H_{1} / L$.

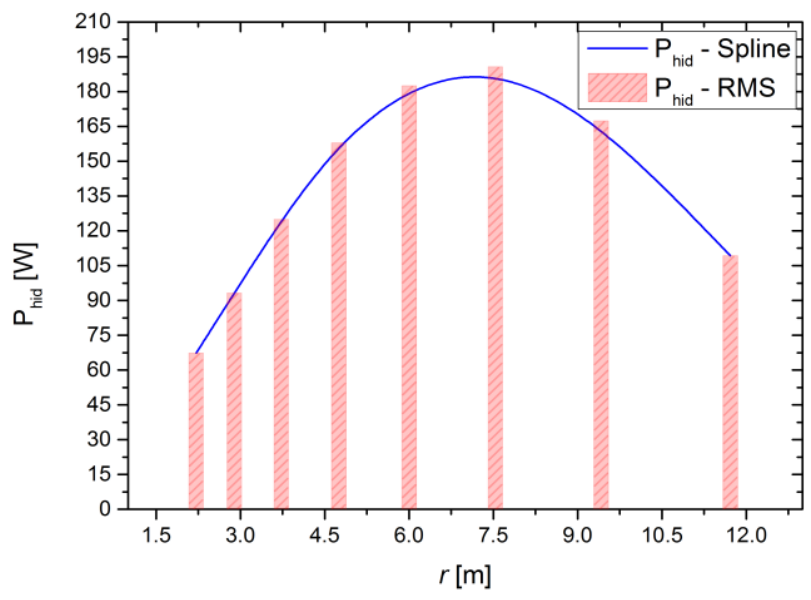

Figura 4: Valores de potência hidropneumática para $r$.

\section{CONCLUSÃO}

No presente trabalho foi realizado um estudo numérico com o objetivo de analisar a influência da região de transição semicircular entre a câmara CAO e a chaminé de um dispositivo conversor de energia das ondas do mar em energia elétrica do tipo Coluna de Água Oscilante (CAO). Para tanto, o método Design Construtal foi usado para definir as diversas variações das geometrias, permitindo definir qual arranjo geométrico que possibilita um melhor desempenho do sistema CAO, possibilitando ainda analisar a influência deste tipo de região de transição sobre o escoamento de ar pelo dispositivo CAO.

A análise dos resultados apresentados mostram que os valores de potência hidropneumática em função de $H_{1} / L, H_{2} / l$ e $r$, respectivamente, se mostram os esperados em comparação com as 
pesquisas realizadas anteriormente por Lima et al. $(2004,2014)[2,14]$, onde somente se variou um grau de liberdade, não os três ao mesmo tempo. A variação da dimensão do raio da transição (r) é responsável pelo aumento de cerca de $64 \%$ na potência hidropneumática, afetando diretamente a configuração geométrica do dispositivo. A variação do grau de liberdade $H_{2} / l$ não se mostra tão evidente, a justificativa para os valores ficarem todos concentrados no mesmo ponto é que para esse grau de liberdade $H_{2}=9,83 \mathrm{~m} \mathrm{e} l=1,64 \mathrm{~m}$ é o maior valor para a altura do tanque e o menor valor para a largura do mesmo, se concentrando a maior quantidade de pressão, essa que influência diretamente no cálculo da potência hidropneumática.

Os resultados obtidos mostram que a máxima potência hidropneumática de 190,61 W é obtida para os graus de liberdade $H_{1} / L=0,1346, H_{2} / l=6$ e $r=7,54 \mathrm{~m}$. O resultado com a menor potência hidropneumática se obteve com a configuração de $H_{1} / L=1,0225, H_{2} / l=5$ e $r=2,13 \mathrm{~m}$, onde se obteve um valor de 57,73 W. Este resultado mostra um ganho de 69,71\% em relação ao caso com menor desempenho de potência hidropneumática.

A aplicabilidade do método Design Construtal para a avaliação geométrica de dispositivos conversores de energia das ondas foi evidenciada, uma vez que os resultados indicam um melhor desempenho do dispositivo. A otimização é feita através da técnica de busca exaustiva. Num paralelo com a Teoria Construtal, é possível avaliar a evolução da configuração geométrica que conduz para o melhor aproveitamento. Portanto, a técnica de Design Construtal se apresenta como uma ferramenta de extrema relevância no desenvolvimento deste tipo de análise.

Para futuras pesquisas, será interessante avaliar outros tipos de forma geométrica na região de transição bem como realizar uma investigação do problema tridimensional associado a este estudo e comparar com outras regiões de transição com geometrias diferentes.

\section{AGRADECIMENTOS}

Os autores agradecem à Universidade Federal do Rio Grande do Sul (UFRGS), à Universidade Federal do Rio Grande (FURG) e ao Instituto Federal de Educação, Ciência e Tecnologia do Paraná (IFPR) pelo apoio e ao Conselho Nacional de Desenvolvimento Científico e Tecnológico (CNPq) pelo suporte financeiro.

\section{REFERÊNCIAS BIBLIOGRÁFICAS}

1. Cruz JP, Sarmento AJ. Energia das ondas: introdução aos aspectos tecnológicos, econômicos e ambientais. Portugal: Instituto do Ambiente; 2004. 65 p.

2. Lima YT, Rocha LA, Plamer CB, Isoldi LA, Santos ED, Gomes MN. Analise numérica com constructal design da forma geométrica na região de transição entre a câmara hidropneumática e a chaminé de um dispositivo do tipo coluna de agua oscilante. Proceedings of the XXXVI Iberian Latin-American Congress on Computational Methods in Engineering; 2015 Nov 22-25; Rio de Janeiro, RJ. p. 1-20, doi:10.20906/CPS/CILAMCE2015-0256.

3. Gomes M. Constructal design de dispositivos conversores de energia das ondas do mar em energia elétrica do tipo coluna de água oscilante [Thesis]. Porto Alegre (RS): Universidade Federal do Rio Grande do Sul; 2014. 168 p.

4. Bejan A, Lorente S. Constructal law of design and evolution: physics, biology, technology, and society. Journal of Applied Physics. 2013 Apr;113(15):01-20, doi: 10.1063/1.4798429.

5. Bejan A, Zane JP. Design in nature: how the constructal law governs evolution in biology, physics, technology and social organization. United States: Doubleday Press; 2012. 304 p.

6. Gomes MN, Olinto CR, Rocha LAO, Souza JA, Isoldi L A. Computational modeling of the air-flow in an oscillating water column dystem. Proceedings of the 20th International Congress of Mechanical Engineering; 2009 Nov 15-20; Gramado, RS. p 1-10.

7. Gomes M, Bonafini BL, Isoldi LA, Nascimento CD, Santos ED, Rocha LA. Two-dimensional geometric optimization of na oscillating water column converter in laboratory scale. Thermal Engineering. 2012 Jun;12(11):30-36.

8. Gomes MN, Isoldi LA, Rocha LA. Two-dimensional geometric optimization of an oscillating water column converter of real scale. Proceedings of the 22nd International Congress of Mechanical Engineering; 2013 Nov 3-7; Ribeirão Preto, SP. p. 1-12. 
9. Lopes N, Sant'anna FS, Gomes MN, Souza JA, Teixeira PR, Santos ED, Isoldi LA, Rocha LA. Constructal design optimization of the geometry of an oscillating water column wave energy converter (OWC-WEC). Proceedings of the Constructal Law Conference; 2012 Dec 01-02; Porto Alegre, RS. p. $1-8$.

10. Oliveira L, Teixeira PR, Santos ED, Isoldi LA. Constructal design applied to the geometric optimization of the hydropneumatic chamber dimension of an oscillating water column wave energy device. Proceedings of the 11th Young symposium on experimental solid mechanics; 2012 May 30-31; Brasov, Romenia. p. 173-178.

11. Espinel MF, Gomes MN, Rocha LA, Santos E, Isoldi LA. Numerical modeling and constructal design applied to the performance improvement of an oscillating water column wave energy converter. Proceedings of the $15^{\text {th }}$ Brazilian Congress of Thermal Sciences and Engineering. 2014 Nov 10-13; Belém, PA. p. 01-10.

12. Espinel MF, Gomes MN, Rocha LA, Santos ED, Isoldi LA. Modelagem computacional e otimização da conversão de energia com constructal design de um dispositivo do tipo coluna de água oscilante. Proceedings of the XXXV Iberian Latin-American Congresso in Computational Methods in Engineering; 2014 Nov 23-26; Fortaleza, CE. p. 01-20.

13. Espinel M. Estudo numérico bidimensional com aplicação de constructal design para a otimização da geometria e da profundidade de submersão de um dispositivo conversor das ondas do mar do tipo coluna de água oscilante [dissertation]. Porto Alegre (RS): Universidade Federal do Rio Grande do Sul; 2015. 85 p.

14. Lima YT, Rocha LA, Isoldi LA, Santos ED, Gomes MN. Análise numérica com constructal design da forma geométrica na região de transição entre a câmara hidropneumática e a chaminé de um dispositivo do tipo coluna de água oscilante. Proceedings of the XXXVI Iberian Latin-American Congress on Computational Methods in Engineering; 2015 Nov 22-25; Rio de Janeiro, RJ. p. 01-20, doi: 10.20906/CPS/CILAMCE2015-0258.

15. Fluent Inc. FLUENT 12.0 theory guide. Canonsburg: SouthPoint Press; 2009. 814 p.

16. Maliska CR. Transferência de calor e mecânica dos fluidos computacional. Rio de Janeiro: LTC-Livros Técnicos e Científicos; 2004. 453 p.

17. Gomes MN, Isoldi LA, Santos ED, Rocha LA. Análise de malhas para geração numérica de ondas em tanques. Proceedings of the VII Congresso Nacional de Engenharia Mecânica; 2012 Aug 01-03; São Luiz, MA. p. 1-10.

18. Liu Z, Hyun B, Hong K. Application of numerical wave tank to OWC air chamber for wave energy conversion. Proceedings of the International Offshore and Polar Engineering Conference; 2008 Jul 0611; Vancouver, BC, Canada. p. 350-356.

19. Ramalhais R. Estudo numérico de um dispositivo de conversão da energia das ondas do tipo coluna de água oscilante (CAO) [dissertation]. Lisboa (PT): Faculdade de Ciências e Tecnologia da Universidade Nova de Lisboa; 2011. 103 p.

20. Conde JM, Gato LM. Numerical study o fair-flow in an oscillanting water column wave energy converter. Renewable Energy. 2008 Feb;12(33):2637-44.

21. Gomes MN, Olinto CR, Rocha LA, Souza JÁ, Isoldi LA. Computacional modeling of a regular wave tank. Thermal Engineering. 2009 Jun;01(08):44-50.

22. McCormick ME. Ocean engineering wave mechanics. New York: John Wiley \& Sons; 1976. 202 p.

23. Dean RG, Dalrymple RA. Water wave mechanics for engineers and scientists. Singapura: World Scientific press; 1991. 353 p.

24. Chakrabarti SK. Handbook of offshore engineering. Estados Unidos: Elsevier, Ilinois; 2005. 661 p.

25. Srinivasan AJ, Saito K. Modeling the disintegration of modulated liquid jets using volume-of-fluid (VOF) methodology. Applied Mathematical Modeling. 2011 Jan;08(35):3710-3730.

26. Patankar SV. Numerical heat transfer and fluid flow. Estados Unidos: McGraw-Hill; 1980. 196 p.

27. Versteeg HK, Malalasekera W. An introduction to computational fluid dynamics. Malaysia: Pearson Press; 2007. 520 p.

28. Horko M. CFD Optimization of an oscillating water column energy converter [dissertation]. Western (AU): University of Western; 2007. 159 p.

29. Liu Z, Hyun B, Jin J. Numerical prediction for overtopping performance of OWEC, Journal of the Korean Society for Marine Environmental Engineering. 2008 Apr;01(11):35-41, doi: 10.1109/OCEANSKOBE.2008.4531009.

30. Gomes M. Modelagem computacional de um dispositivo coluna d'água oscilante de conversão de energia das ondas do mar em energia elétrica [dissertaton]. Rio Grande (RS): Universidade Federal do Rio Grande; 2010. 206 p.

31. Mavriplis DJ. Unstructured grid techniques. Annual Reviews Fluid Mechanics. 1997 Jan;(29):473-514, doi: 10.1146/annurev.fluid.29.1.473. 
32. Marjani AE, Castro F, Bahaji M, Filali B. 3D Unsteady flow simulation in na OWC wave converter plant. Proceedings of the International Conference on Renewable; 2006 Apr 05-06; Balearic Island, Spain. p. 01-20.

33. Dizadji N, Sajadian SE. Modeling and optimization of the chamber of OWC system. Energy, 2011 April;5(36):2360-66.

34. Zhang Y, Zou QP, Greaves D. Air-water two-phase flow modelling of hydrodynamic performance of an oscillating water column device. Renewable Energy. 2012 May;10(41):159-70. 